\title{
X-ray measurement of residual stresses and texture development during a rolling sequence of zirconium alloy cladding tubes - influence of plastic anisotropy on mechanical behaviour
}

\author{
David Gloaguen, Jamal Fajoui, Emmanuel Girard and Ronald Guillén
}

GeM, Institut de Recherche en Génie Civil et Mécanique, Université de Nantes, CNRS UMR 6183, 37 Boulevard de l'Université, BP 406,44 602 Saint-Nazaire, France. Correspondence e-mail: david.gloaguen@univ-nantes.fr

\begin{abstract}
Texture and residual stress analysis using X-ray diffraction have been carried out on zirconium alloy cladding tubes after cold pilgering, an industrial mechanical process. The final rolling pass has been completely characterized by X-ray diffraction. An interpretation of the effect of intergranular stresses on the development of analysed stress has been made using a modified elastoplastic self-consistent model in order to account for the effect of the high intrinsic plastic anisotropy of hexagonal close-packed crystals. The contribution and magnitude of the first- and second-order residual stresses were correctly evaluated using information from the model. The main features of the rolling sequence were qualitatively reproduced by the simulations, considering prismatic slip as the main active deformation mode in this alloy under large strain.
\end{abstract}

\section{Introduction}

The fabrication of components with appropriate structural properties has always been a real challenge to thermomechanical processing. One example is the selected component of this study, M5 alloy cladding tubes manufactured by cold pilgering. Zirconium alloys such as M5 are used extensively in various types of fission reactors, and indeed the development of $\mathrm{Zr}$ alloys is essentially due to the nuclear industry, where they have been regarded as proven structural materials (Linga Murty \& Charit, 2006). Cold pilgering is a tube-forming operation (see Fig. 1), where the inner radius and wall thickness are both progressively reduced between a fixed axisymmetric mandrel and forward- and backwardrolling grooved dies (Montmitonnet et al., 2002). The process is quasi-periodic, consisting of several tens of 'strokes', between which the tube is turned by about $51^{\circ}$ and advanced a small distance. A metal particle thus progresses by a few millimetres per stroke down the $300 \mathrm{~mm}$-long deformation zone of the mandrel (it takes $80-100$ strokes before the deformation is completed). The process consists of a sequence of three rolling passes. After each pass, the tube is heat-treated at a certain annealing temperature which is sufficient to induce recrystallization, except for the final heat treatment which is done simply for relief of stresses.

Owing to their hexagonal close-packed (h.c.p.) structure, $\mathrm{Zr}$ alloys exhibit highly anisotropic plastic properties at mesoscopic (grain) and macroscopic levels with various active deformation modes. These properties and the crystallographic texture explain the appearance and development of important residual stresses during mechanical treatment. These stresses are termed intergranular or second-order stresses. They depend on initial and induced crystallographic textures. The engineering consequences of second-order stresses with strong texture can be severe: the texture and stresses at a given step in the fabrication process will influence the formability with which the next mechanical process might be realized. The texture of the material will have a significant effect on its performance in service because irradiation creep, yield strength, stress, corrosion and cracking resistance, for example, are strong functions of texture. During processing,

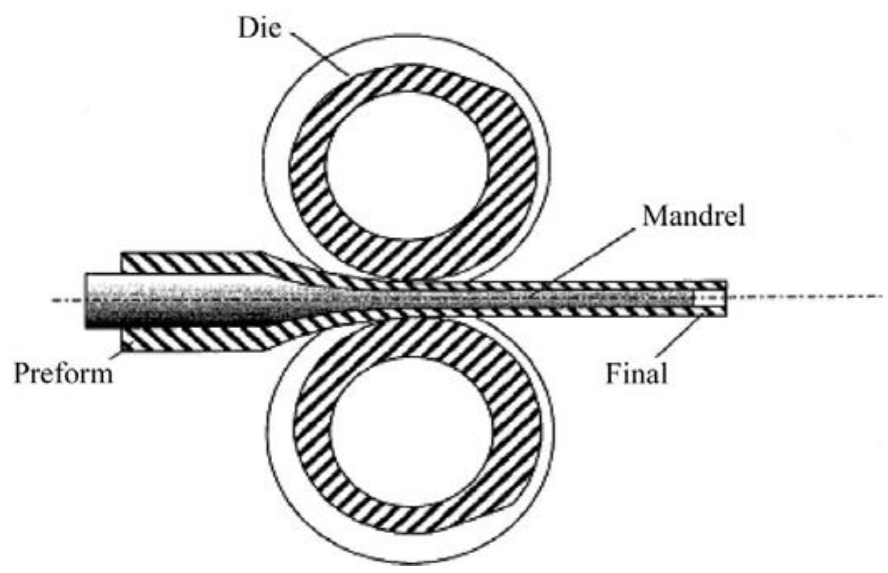

Figure 1

Schematic view of cold pilgering. 
excessive internal stresses can also induce defects such as transverse cracks or surface damage (Girard et al., 2001; Linga Murty \& Charit, 2006).

A thorough mechanical analysis of the development of stresses and texture during the different stages of fabrication is necessary to optimize processing routes and predict lifetimes of reactor components. The macro- and mesoscopic stresses that are generated by thermo-mechanical treatment have complex origins, but can be interpreted thanks to polycrystalline models such as the self-consistent (SC) approach (Clausen et al., 1998; Matthies et al., 2001; Gloaguen et al., 2007; Proust et al., 2007; Xu et al., 2008). Polycrystalline models are typically evaluated by their ability to simulate mechanical behaviour and they lead to an understanding of the relative contributions of different deformation modes to the macroscopic strain.

Hexagonal materials are characterized by a wide variety of possible deformation systems. It is necessary to know both the texture and the deformation mechanisms to be able to obtain a good model of these mechanical properties. This requires, in particular, a proper knowledge of the deformation mechanisms with their corresponding critical resolved shear stresses (CRSSs). Only a few data are available for CRSS in the literature. Moreover, CRSS generally depends significantly on the content of the alloying elements. In spite of this very basic knowledge, many authors have tried to model the mechanical properties of h.c.p. materials after large deformations. In these cases, the approach consists of finding the set of material parameters offering the best agreement with crystallographic texture by applying a polycrystalline model. Realistic simulations of deformation texture development in $\mathrm{Zr}$ alloys can be carried out using the viscoplastic SC model (Tomé et al., 1991; Castelnau et al., 2001; Kaschner et al., 2006). The coldpilgering texture of $\mathrm{Zr}$ alloys has also been studied with this kind of approach (Lebensohn et al., 1992; Girard et al., 2001). A comparison of simulations and experimental texture (obtained from the final cladding tube) shows that prismatic slip is probably the most active system for this process.

In the present work, a different approach is proposed to validate the accuracy and relevance of the theoretical model for h.c.p. materials undergoing a large deformation. Characterization by diffraction of both texture and residual stresses has been used simultaneously to evaluate polycrystalline models. A direct comparison between experimental observations (X-ray diffraction) and the predictions of an elastoplastic self-consistent (EPSC) model has been made in order to interpret the residual strain (or stress) and the development of texture, and hence to deduce the active deformation mechanisms during mechanical processing. This comparison allows a better understanding and interpretation of diffraction and mechanical results.

The aim of the present study is to analyse and understand the evolution of anisotropic elastoplastic behaviour during the fabrication of $\mathrm{Zr}$ alloy tubes. A modified EPSC model was used to predict the behaviour of $\mathrm{Zr}$ alloy cladding tubes during the final rolling pass. This approach is well suited to studying anisotropic materials with multiple slip and twinning modes because it allows for different deformation modes depending on the relative orientation between the grain and the average medium. X-ray diffraction results (texture and residual stresses) obtained from the different states provide an accurate experimental basis for determining the appropriate model parameters and finding a realistic combination of deformation systems. The theoretical stresses are compared with the experimental results obtained by X-ray diffraction. In this paper, we aim to provide a better understanding of the influence of different deformation modes and their contributions to polycrystalline plasticity during a real industrial mechanical process.

To the best of our knowledge, characterization by diffraction of texture and/or residual stresses has been made only after each different sequence of rolling passes for $\mathrm{Zr}$ alloy cladding tubes (Lebensohn et al., 1992; Guillén et al., 1998; Kapoor et al., 2002; Kiran Kumar et al., 2004; Linga Murty \& Charit, 2006). No information on the development of texture and stresses during rolling passes is available in the literature. In order to obtain an extensive set of experimental data on the evolution of residual strain and texture, the final rolling pass has been completely characterized by X-ray diffraction in the transition area between the raw tube and the finished one (see Fig. 1).

\section{Experimental procedure}

The analysis was performed for the final cold-pilgering pass. Before this stage, the tubes have been submitted to a $973 \mathrm{~K}$ recrystallization treatment, and the dimensions of the forming M5 tubes are $17.8 \mathrm{~mm}$ external diameter and $2 \mathrm{~mm}$ thickness. The dimensions after forming (195\% total strain) are 8.37 and $0.6 \mathrm{~mm}$, respectively. The chemical composition is $(\mathrm{wt} \%$; balance $=\mathrm{Zr}) \mathrm{Nb}(1.0)$, Fe (0.035) and $\mathrm{O}(0.13)$.

To observe the evolution of the mechanical state at each step of the tube-forming operation, the rolling was interrupted. Truncated samples were obtained for the X-ray diffraction study and analyses were made in the transition area between the preform tube ( $0 \%$ total strain and $z=0 \mathrm{~mm})$ and the finished one $(195 \%$ total strain and $z=300 \mathrm{~mm})$. We analysed the texture and residual stresses at different total strains of 0, 21, 51, 71, 95, 124, 159, 189, 193 and 195\%. Fig. 2 shows the different measurement points and the relation between the axial position $z$ along the rolling direction and the total macroscopic strain in the transition area.

\subsection{Crystallographic texture analysis}

X-ray diffraction (XRD) analysis was performed using a Seifert XRD 3003 PTS four-circle diffractometer. $\mathrm{Cu} \mathrm{K \alpha}$ radiation was used as the $\mathrm{X}$-ray source $(40 \mathrm{kV}, 40 \mathrm{~mA}$ with $\mathrm{Ni}$ filter). The X-ray beam output collimator had a diameter of $0.5 \mathrm{~mm}$. The diffraction peaks were recorded with a positionsensitive detector (PSD). Incomplete pole figures (PF) were achieved on a $5 \times 5^{\circ}$ grid, with tilt and azimuth angles in the ranges $0-60$ and $0-360^{\circ}$, respectively. For each experimental direction, the diffraction pattern was adjusted, using a 
nonlinear least-squares analysis and assuming pseudo-Voigt peak profiles (i.e. a linear combination of Gaussian and Lorentzian functions; Guinebretière, 2007), in order to evaluate background noise and obtain peak intensities. The study of samples with a curved geometry requires not only accurate positioning of the samples but also taking into account the geometric effects induced by XRD directional features. A theoretical model based on a ray-tracing method was used to take into account the geometric effects that modify the collected intensities; more details of this approach are given by Guillén et al. (1999). Correction coefficients accounting for the 'geometric texture' of the samples, as well as absorption corrections, were calculated for each measurement point. The results obtained were used to adjust the experimental pole figures. Fig. 3 shows the experimental $\{0002\}$ PF for the finished tube before and after correction. Poles densities in the RD-ND (RD is the rolling direction and ND is the normal direction) plane are affected, showing the effect of the tilt angle $\psi$. An increase in the pole maximum density for the corrected pole figure compared with the uncorrected one is observed. The maximum is in the TD-ND (TD is the transverse direction) plane, making a $30^{\circ}$ angle with the normal direction of the tube. With this method, some surface effects, not detected on flattened samples, could be revealed in the cylindrical samples. An underestimation of the pole density on a significant part of the pole figure therefore involves an underestimation of the texture sharpness and an error in the anisotropy evaluation of the material properties. After this geometric correction, an orientation distribution function (ODF) was calculated, with experimental PF $\{0002\},\{10 \overline{1} 1\}$, $\{11 \overline{1} 0\}$ and $\{10 \overline{1} 3\}$, with the help of the WIMV algorithm implemented in the BEARTEX program package (Wenk et al., 1998).

\subsection{Determination of surface residual stresses}

We present briefly the principles and different assumptions of residual stress evaluation by XRD and the key role played by plastic anisotropy properties in the interpretation of the

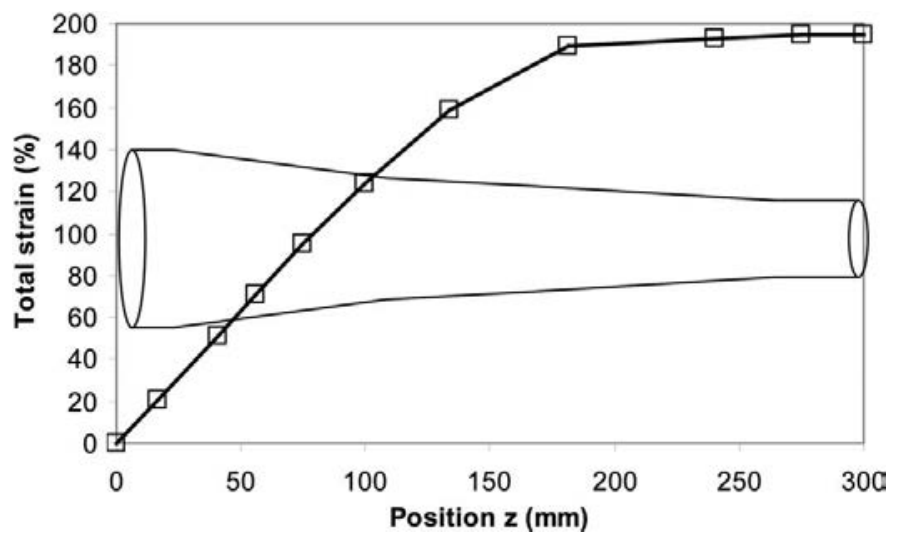

Figure 2

Correspondence between the axial position $z$ along the rolling direction and the total macroscopic strain. The different measurement points are marked on the figure as squares. data. More details can be found elsewhere (Baczmanski et al., 2009; Hauk, 1997; Noyan \& Cohen, 1987; Ortiz \& Pochettino, $1996 a, b)$.

The residual stress field in a polycrystalline material depends on both the thermomechanical history of the sample and its internal structure. The usual classification of stresses when dealing with diffraction methods is based on the length scale (Macherauch et al., 1973). Residual stresses of type I, $\sigma^{\mathrm{I}}$ (first-order stresses), represent the average residual stresses $\sigma(r)[\sigma(r)$ denotes the local residual stress at a position $r$ in the sample] acting within all crystallites in the considered volume $V$, for example the volume exposed to the X-ray beam. These stresses result from long-range strain incompatibilities introduced by strain or temperature gradients in the manufacturing process.

$$
\sigma^{\mathrm{I}}=\frac{1}{V} \int_{V} \sigma(r) \mathrm{d} V
$$

Residual stresses of type II, $\sigma^{\mathrm{II}}$ (second-order stresses), within a crystal represent the mean deviation from the macroscopic residual stress level.

$$
\sigma^{\mathrm{II}}=\frac{1}{V_{g}} \int_{V_{g}}\left[\sigma(r)-\sigma^{\mathrm{I}}\right] \mathrm{d} V=\sigma^{g, r}-\sigma^{\mathrm{I}} .
$$

$V_{g}$ denotes the volume of a grain $g$ and $\sigma^{g, r}$ the residual stresses at the grain level, which are different from macroscopic stresses; this difference is due to deformation misfits between neighbouring grains and temperature effects linked to the anisotropy of the elastic constants, thermal expansion coefficients or/and plastic flow at the grain level.

In XRD, the definition of second-order stresses is different and has been adapted to this method (Hauk, 1997). These stresses must be understood as the average over the group of crystallites contributing to the measurement, i.e. the diffracting volume $V_{\mathrm{d}}$.

$$
\sigma^{\mathrm{II}}=\frac{1}{V_{\mathrm{d}}} \int_{V_{\mathrm{d}}}\left[\sigma(r)-\sigma^{\mathrm{I}}\right] \mathrm{d} V .
$$

Residual stresses of type III (microstresses or third-order stresses), $\sigma^{\mathrm{III}}(r)$, are the local deviation of the residual stresses

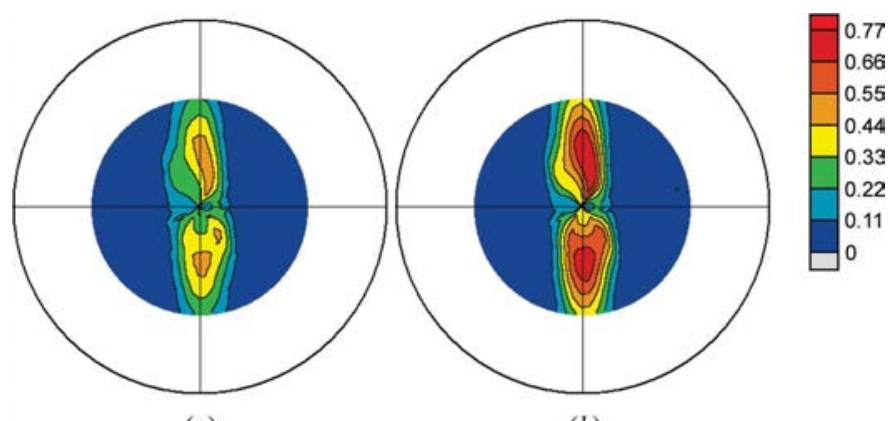

(a)

(b)

Figure 3

Experimental $\{0002\}$ incomplete PF of the final tube $(a)$ before and $(b)$ after geometric correction. 
within an individual crystallite from its average residual stress. These stresses are caused by, for example, voids, second-phase particles, precipitates and dislocations in the crystal lattice.

Diffraction experiments provide information about the mechanical behaviour of groups of polycrystalline grains in the near-surface volume during thermomechanical treatment. Using X-ray diffraction as an analytical tool, the change in the lattice parameters due to the occurrence of strains (which induce displacement of the diffraction peak) is measured to determine the residual stresses defined above (Dölle, 1979; Noyan \& Cohen, 1987; Hauk, 1997). After the forming process, residual stresses can be generated by inhomogeneous plastic deformation on two length scales, one given by the size of the component and the other by the size of the grains forming the polycrystalline aggregate. Inhomogeneity on the sample length scale gives the macroscopic stress field or firstorder stress, while inhomogeneity on the grain size scale gives the intergranular or second-order stresses. The two are superimposed, and XRD measurements give a combination of first- and second-order stresses (Ortiz \& Pochettino, 1996a). Taking into account the definition of microstresses, it should be emphasized that their mean value is zero within each grain and, as a consequence, within the diffracting volume. These stresses introduce variations in the interplanar spacing around its mean value, which induce only a broadening of the diffraction peaks and not a shift, in contrast with the first- and the second-order stresses.

In order to determine first-order residual stresses, intergranular stresses must thus be subtracted from the given stress field. The important question is how to obtain the macroscopic strain from the measured strain in a hexagonal component observed with the help of a specific $\{h k i l\}$ plane when we have a superposition of both macroscopic and important intergranular contributions. A method for the quantitative evaluation of second-order stresses developed by Baczmanski et al. (1994) has been used and extended to h.c.p. materials. This method (summarized below) allows the separation of macrostresses and second-order stresses created as a result of the plastic anisotropy.

For a particular direction of the scattering vector defined by the angles $\varphi$ and $\psi$, the measured strain can be expressed by

$$
\langle\varepsilon(\varphi, \psi, h k i l)\rangle_{V_{\mathrm{d}}}=F_{i j}(\varphi, \psi, h k i l) \sigma_{i j}^{\mathrm{I}}+\left\langle\varepsilon^{\text {IIplasticity }}(\varphi, \psi, h k i l)\right\rangle_{V_{\mathrm{d}}} .
$$

[More details about the establishment of this relation are given by Baczmanski et al. $(1994,2009)$ and Gloaguen et al. (2004).] $\sigma^{\mathrm{I}}$ is the first-order residual stress tensor, $F_{i j}(\varphi, \psi, h k i l)$ are the diffraction elastic constants for the $\{h k i l\}$ reflection and $\langle\ldots\rangle_{V \mathrm{~d}}$ denotes the average over the diffracting grains for the $\{h k i l\}$ reflection. Thus, $\left\langle\varepsilon^{\text {IIplasticity }}(\varphi, \psi, h k i l)\right\rangle_{V_{\mathrm{d}}}$ is the average plastic intergranular strain over the volume of diffracting grains for a given $\{h k i l\}$ reflection and for a particular orientation of the scattering vector. It represents the elastic strain caused by the misfit of the plastic strain in the grains (contributing to the measured diffraction peak) in relation to the surrounding matrix. As a consequence of the anisotropic properties of a single crystal, the response of the grains within the aggregate is different from that of isolated crystals, and this is responsible for the development of internal stresses. Plastic intergranular strains (and stresses) are induced during plastic deformation because full accommodation of the shape of each grain is prevented by neighbouring grains, since the plastic strain varies from one crystallite to another. The term $F_{i j}(\varphi, \psi, h k i l) \sigma_{i j}^{\mathrm{I}}$ in equation (4) is linked to the second-order stresses caused by the anisotropy of the elastic properties. Consequently, the elastic response of each grain to the residual macroscopic stress $\sigma^{\mathrm{I}}$ varies according to the grain orientation relative to neighbouring grains. The term $F_{i j}(\varphi, \psi, h k i l) \sigma_{i j}^{\mathrm{I}}$ is the additional elastic strain caused by the residual macroscopic stress due to the processing heterogeneity averaged over the volume of diffracting grains for a given $\{h k i l\}$ reflection and for a particular orientation of the scattering vector, through the determination of the diffraction elastic constants $F_{i j}(\varphi, \psi, h k i l)$.

Equation (4) clearly shows that the measured strain cannot be identified with the macroscopic strain if the material presents plastic anisotropic properties. The presence of intergranular strain after a mechanical manipulation influences the measured strain. Generally, the interpretation of experimental data is based on the unjustified assumption that $\left\langle\varepsilon^{\text {IIplasticity }}(\varphi, \psi, h k i l)\right\rangle_{V_{\mathrm{d}}}=0$. In our study, we propose to quantify the importance of these intergranular stresses and show their influence on the interpretation of the experimental results.

With Baczmanski's method, some information obtained from the scale transition method has been used. The mesocopic elastic strain tensor for each grain after the rolling process can be predicted by the EPSC model. So, the average $\left\langle\varepsilon^{\text {IIth }}(\varphi, \psi, h k i l)\right\rangle_{V_{\mathrm{d}}}$ ('th' means theoretical model) strain for the volume of diffracting grains can be calculated (see $\$ 3$ ). As was shown by Baczmanski et al. (1994), the predicted values of $\left\langle\varepsilon^{\text {IIth }}(\varphi, \psi, h k i l)\right\rangle_{V_{\mathrm{d}}}$ depend on the hardening and relaxation processes, which cannot be accurately taken into account in models. Only the variation in (and not the magnitude of) the residual strains of plastic origin (in relation to $\varphi$ and $\psi$ ) can be correctly predicted. Baczmanski et al. (1994) proposed the introduction of an unknown scaling factor $q$, which is inserted in order to find the real amplitude of elastic strains (or stresses) of plastic origin.

So, the second term in equation (4) is assumed to be approximated by

$$
\left\langle\varepsilon^{\text {IIplasticity }}(\varphi, \psi, h k i l)\right\rangle_{V_{\mathrm{d}}}=q\left\langle\varepsilon^{\text {IIth }}(\varphi, \psi, h k i l)\right\rangle_{V_{\mathrm{d}}} .
$$

Using a scale transition model, we can predict the values of the residual stress and strain tensor for every crystalline orientation and calculate the average strain $\left\langle\varepsilon^{\mathrm{Ith}}(\varphi, \psi, h k i l)\right\rangle_{V_{\mathrm{d}}}$ for the diffracting volume. For known values of $F_{i j}(\varphi, \psi, h k i l)$, the theoretically predicted strain $\left\langle\varepsilon^{\text {IIth }}(\varphi, \psi, h k i l)\right\rangle_{V_{\mathrm{d}}}$ and the measured deformation $\left\langle\varepsilon^{\mathrm{II}}(\varphi, \psi, h k i l)\right\rangle_{V_{\mathrm{d}}}$, the other quantities from equation (4) (the unknowns are $q$ and the first-order stress tensor) can be determined using a nonlinear fitting 
procedure, to give a complete description of the stress (and strain) field in a plastically deformed polycrystalline material.

We have determined the evolution of internal stresses due to plastic anisotropy in the deformed sample for different values of total macroscopic strain (Fig. 2). These experiments were carried out on a Seifert XRD 3003 PTS diffractometer with $\mathrm{Cr} K \alpha$ and $\mathrm{Cu} K \alpha$ radiation. An $\Omega$ goniometric assembly with a PSD was used. Three plane families were studied with $\mathrm{Cr}$ radiation, $\{10 \overline{1} 4\}$ at $2 \theta=156.7^{\circ},\{20 \overline{2} 2\}$ at $2 \theta=137.2^{\circ}$ and $\{0004\}$ at $2 \theta=125.6^{\circ}$, and three others with $\mathrm{Cu}$ radiation, $\{20 \overline{2} 5\}$ at $2 \theta=136.8^{\circ},\{30 \overline{3} 2\}$ at $2 \theta=123.0^{\circ}$ and $\{21 \overline{3} 3\}$ at $2 \theta=$ $117.8^{\circ}$. Spectra were recorded for 16 tilt angles $\psi$ varying between -48 and $45^{\circ}$ and for one azimuthal angle $\varphi=0^{\circ}$, which corresponds to the rolling direction. For the $\{30 \overline{3} 2\}$ plane, only the final tube was analysed. For the other points, the analyses exhibit significant errors for the stress values, linked to a weak peak intensity for several $\left(0^{\circ}, \psi\right)$ orientations. The X-ray beam output collimator had a diameter of $0.5 \mathrm{~mm}$, and the errors due to geometry effects are weak with an X-ray beam of this diameter (Dionnet et al., 1999). The set of diffraction peaks obtained for all the tilt angles was fitted simultaneously using a pseudo-Voigt function, which takes into account the $K \alpha_{1}-K \alpha_{2}$ contribution. The centroid of the fitted diffraction line was taken as the peak position. For each diffraction peak, the background was fitted by a polynomial function. The diffraction elastic constants $F_{i j}(\varphi, \psi, h k i l)$ were calculated theoretically using an elastic SC model (Hauk, 1997). The influence of the texture on these constants was taken into account by weighting the single-crystal elastic constants with the texture function (1000 grains). A stress tensor $\sigma_{i j}^{\exp }(h k i l)$ (exp denotes experimental) is calculated by the relation

$$
\langle\varepsilon(\varphi, \psi, h k i l)\rangle_{V_{\mathrm{d}}}=F_{i j}(\varphi, \psi, h k i l) \sigma_{i j}^{\exp }(h k i l) .
$$

These stresses vary with the $\{h k i l\}$ reflection because of plastic anisotropy. In this case, the second-order stresses of plastic origin $\left[\left\langle\varepsilon^{\text {IIplasticity }}(\varphi, \psi, h k i l)\right\rangle_{V_{\mathrm{d}}}\right.$ in equation (4)] are presumed to be 0 . In the second step, the complete equation (4) is used with an EPSC model to determine both first- and second-order stresses. As the samples were single phased and the penetration depth of the X-rays was small, it was assumed that $\sigma_{33}=0$ in order to calculate the stress tensor (biaxial stress state in the measured region).

\section{Scale transition self-consistent model}

In this study, an EPSC model is used to predict plastic deformation of the M5 alloy cladding tube during the last cold-pilgering pass at the different scales of the material. More details concerning this approach are given by Kröner (1961), Hill (1965), Hutchinson (1970), Lipinski \& Berveiller (1989) and Gloaguen et al. (2007). This model was used to interpret the experimental results and to determine the CRSS, the mechanical state at the meso- and macroscopic levels after the forming process, the crystallographic texture and the strain- hardening parameters. Briefly, the EPSC model is based on the following principal assumptions:

(a) Each grain $g$ is considered as a spherical inclusion embedded in a homogeneous effective medium (HEM) having the average properties of the aggregate.

(b) The mesoscopic stress $\boldsymbol{\sigma}^{g}$ and the mesoscopic total strain $\boldsymbol{\varepsilon}^{g}$ in the inclusion are homogeneous. An additive decomposition of the strain rate $\boldsymbol{\varepsilon}^{g}$ on the elastic (e) and plastic (p) parts is used: $\boldsymbol{\varepsilon}^{g}=\boldsymbol{\varepsilon}^{g \mathrm{e}}+\boldsymbol{\varepsilon}^{g \mathrm{p}}$.

(c) The overall response of the material is determined by averaging the mesoscopic terms $\boldsymbol{\sigma}^{g}$ and $\boldsymbol{\varepsilon}^{g}$ over a representative set of orientations.

(d) The macroscopic stress $\boldsymbol{\Sigma}$ and macroscopic strain $\mathbf{E}$ are considered uniform. The properties of the medium are initially undetermined and are solved by iterations. Each grain is treated as an inhomogeneity with elastic (c), slip and twin mechanisms characteristic of a single crystal.

(e) The texture is known and the polycrystal is represented by a weighted discrete distribution of orientations [Euler angles $\left(\varphi_{1} \varphi \varphi_{2}\right)$ ]. Lattice rotation (reorientation by slip and twinning) and texture changes are incorporated in the model.

An incremental EPSC scheme was used to simulate the mechanical loading. At each strain increment, the nonlinear behaviour is approximated by the tangent behaviour. The stress and strain rates, $\dot{\boldsymbol{\sigma}}^{g}$ and $\dot{\boldsymbol{\varepsilon}}^{g}$, of each grain are linearly linked by an elastoplastic tensor $\ell$, which accounts for the plastic and elastic anisotropy of the grains and the hardening effects of the deformation systems during plasticity.

The main modification, which is briefly discussed here, concerns the selection of active slip systems. The new algorithm is much faster and resolves the problem of the ambiguous selection of slip systems. Plastic flow can take place in a grain when the Schmid criterion is verified, i.e. slip (or twinning) occurs if the resolved shear stress $\tau^{t}$ on a system $t$ is equal to the critical value $\tau_{\mathrm{c}}^{t}$, depending on the hardening state of the slip system. This necessary condition is insufficient, and the complementary condition, which states that the increment of the resolved shear stress must be equal to the incremental rate of the CRSS, has to be checked simultaneously. The resolved shear stress is defined as the projection of the mesoscopic stress tensor $\sigma^{t}$ on the considered deformation system. In the small-strain formulation, one has

$$
\tau^{t}=\mathbf{R}^{t}: \boldsymbol{\sigma}^{t}=\tau_{\mathrm{c}}^{t} \text { and } \dot{\boldsymbol{\tau}}^{t}=\mathbf{R}^{t}: \dot{\boldsymbol{\sigma}}^{t}=\dot{\boldsymbol{\tau}}_{\mathrm{c}}^{t},
$$

where $\mathbf{R}^{t}$ is the Schmid tensor on a system $t . \mathbf{A}: \mathbf{B}$ denotes the double scalar product $A_{i j k l} B_{k l m n}$ using the Einstein summation convention.

Recently, Lorrain and co-workers have proposed a new formulation [see, for example, Lorrain et al. (2005) and Franz et al. (2009)]. The accuracy of the simulations was also evaluated at the meso- and macroscopic levels by referring to mechanical experiments (tensile tests, neutron diffraction; Gloaguen et al., 2006). Based on the work of Lorrain et al., the slip rate on a system $t$ can be expressed as

$$
\dot{\gamma}^{t}=M^{t}\left(\tau^{t}, \boldsymbol{\tau}_{\mathrm{c}}^{t}\right) \dot{\tau}^{t} .
$$


The slip rate is linked to the resolved shear stress rate through a function $M^{t}$, a hardening parameter given by (Lorrain et al., 2005)

$$
\begin{aligned}
M^{t}= & \frac{1}{H^{t t}}\left(\frac{1}{2}\left\{1+\tanh \left[k_{0}\left(\frac{\boldsymbol{\tau}^{t}}{\boldsymbol{\tau}_{\mathrm{c}}^{t}}-1\right)\right]\right\}\right) \\
& \times\left\{\frac{1}{2}\left[1+\tanh \left(k_{1} \dot{\boldsymbol{\tau}}^{t}\right)\right]\right\}\left\{\frac{1}{2}\left[1+\tanh \left(k_{1} \boldsymbol{\tau}^{t}\right)\right]\right\},
\end{aligned}
$$

where $k_{0}$ and $k_{1}$ are numerical parameters. $H^{t t}$ is the selfhardening parameter described by the hardening matrix $H^{\text {th }}$ (i.e. the matrix reflecting the interactions between deformation systems; Gloaguen et al., 2007). The hyperbolic tangent function tanh was tested and used in equation (9) because it permits the reproduction of the mechanical and hardening behaviours.

The tensor $\ell$ for a grain $g$ can be written as

$$
\ell=\mathbf{c}-\sum_{t} \sum_{h} \mathbf{c}: \mathbf{R}^{t}\left(\delta_{t h}+M^{t} \mathbf{R}^{t}: \mathbf{c}: \mathbf{R}^{h}\right)^{-1} M^{h} \mathbf{R}^{h}: \mathbf{c},
$$

where $\mathbf{c}$ is the elastic stiffness of the single crystal and $\delta_{t h}$ is the Kronecker delta.

At the macroscopic level, the relation between the macroscopic stress rate $\dot{\boldsymbol{\Sigma}}$ and strain rate $\dot{\mathbf{E}}$ is given by a similar relation with the introduction of a tensor $\mathbf{L}$,

$$
\dot{\boldsymbol{\Sigma}}=\mathbf{L}: \dot{\mathbf{E}}
$$

where $\mathbf{L}$ is the macroscopic tangent modulus for the fictional average homogeneous medium. The local strain and stress rates can be obtained classically through the localization $\mathbf{A}$ and concentration $\mathbf{B}$ tensors with the 'one-site' self-consistent approximation (Lipinski \& Berveiller, 1989)

$$
\begin{aligned}
\dot{\boldsymbol{\varepsilon}}^{g} & =\left(\mathbf{I}+\mathbf{S}^{\mathrm{esh}}: \mathbf{L}^{-1}: \Delta \ell\right)^{-1}: \dot{\mathbf{E}}=\mathbf{A}: \dot{\mathbf{E}}, \\
\dot{\boldsymbol{\sigma}}^{g} & =\ell:\left(\mathbf{I}+\mathbf{S}^{\mathrm{esh}}: \mathbf{L}^{-1}: \Delta \ell\right)^{-1}: \mathbf{L}^{-1}: \dot{\boldsymbol{\Sigma}} \\
& =\ell: \mathbf{A}: \mathbf{L}^{-1}: \dot{\boldsymbol{\Sigma}}=\mathbf{B}: \dot{\boldsymbol{\Sigma}},
\end{aligned}
$$

where $\Delta \ell=\ell-\mathbf{L}$ and $\mathbf{S}^{\text {esh }}$ is the Eshelby tensor.

The volume averages of the local stress and strain tensors must coincide with the overall strain and stress. After some algebraic calculations, these conditions give the overall elastoplastic tensor $\mathbf{L}$ as a weighted average of the mesoscopic tensor $\ell$

$$
\mathbf{L}=\left\langle\ell:\left(\mathbf{I}+\mathbf{S}^{\mathrm{esh}}: \mathbf{L}^{-1}: \Delta \ell\right)^{-1}\right\rangle .
$$

Equation (14) is a nonlinear implicit equation because $\mathbf{S}^{\text {esh }}$ depends on the unknown $\mathbf{L}$ in the framework of EPSC theory. The Eshelby tensor is calculated by an integral equation (Lipinski \& Berveiller, 1989) that takes the plastic anisotropy fully into account. This equation is solved iteratively. Once $\mathbf{L}$ is known, then by specifying an overall stress or strain increment the rate of strain at the grain level, $\dot{\boldsymbol{\varepsilon}}^{g}$, can be determined and other variables characterizing grain evolution and depending on $\dot{\boldsymbol{\varepsilon}}^{g}$ can be calculated. The calculations are performed up to a given total strain $\mathbf{E}$ and then an elastic unloading of the sample is performed in the last step $(\Sigma \rightarrow 0)$. The mesocopic elastic strain tensor $\boldsymbol{\varepsilon}^{g e}$ is determined for each grain $g$ when $\Sigma=0$. Finally, $\left\langle\varepsilon^{\text {IIth }}(\varphi, \psi, h k i l)\right\rangle_{V_{\mathrm{d}}}$ is calculated for each analysed plane [see $\$ 2.2$, equation (5)].

\section{Experimental results}

\subsection{Evolution of crystallographic texture}

Fig. 4 shows the development of texture during the different steps of the tube-rolling process. The initial recrystallized tube exhibits a basal maximum with a preferential alignment parallel to the ND, with a slight spread of approximately $\pm 40^{\circ}$ towards the TD. The formation of this maximum can be explained by the reorientation towards the ND of the ND-TD and ND-RD maxima appearing in the tube before the recrystallization after the second rolling step. The heat treatment leads to a noticeable decrease of the bimodal distribution of the intensity maxima in the ND-TD plane. With plastic deformation, texture evolution is very gradual for this kind of forming process. During the rolling step, prismatic poles are redirected towards the RD. The intensity varies from 2.0 to 9.41 for the final tube. In the final state, the basal pole figure shows intensity maxima in the NT-TD plane at about $30^{\circ}$ of the ND axis. The $c / a$ ratio of this alloy and the deformation path lead to this bimodal texture. More precisely, the ratio

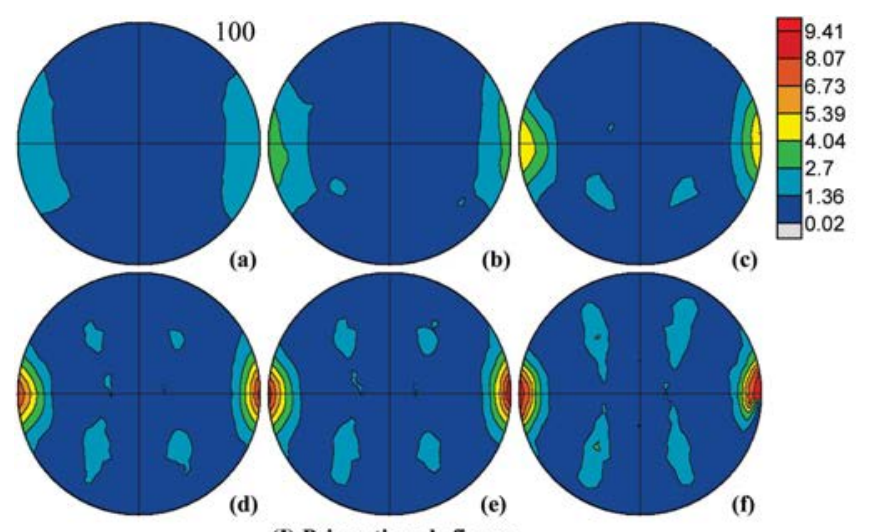

(I) Prismatic pole figures
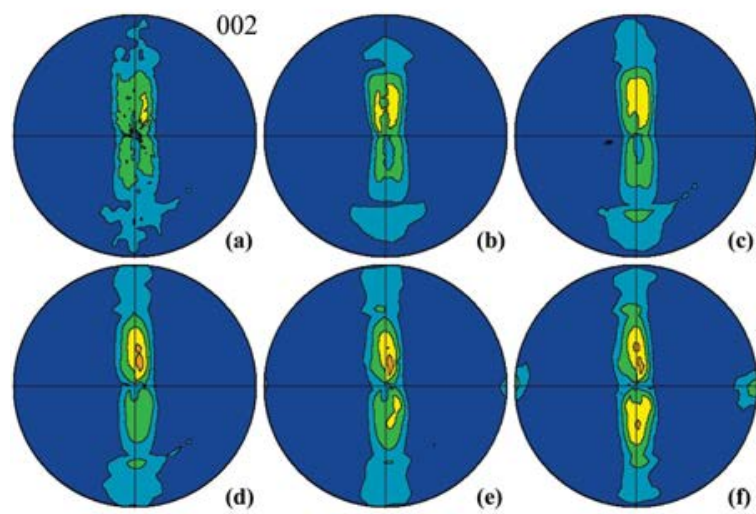

(II) Basal pole figures

Figure 4

(I) Experimental prismatic and (II) basal pole figures, recalculated using the WIMV method, at (a) $0 \%$ (initial texture), (b) $21 \%,(c) 51 \%,(d)$ $124 \%,(e) 159 \%$ and $(f) 195 \%$ total macroscopic strain. 
between thickness reduction and tube diameter reduction has an important influence on tube texture. The tube is mainly reduced in thickness, whereas the compressive forces in the tangential direction are comparatively small. In this case, the basal planes will tend to have a radial orientation because they follow the real direction of the compressive force (Tenckhoff, 1988).

\subsection{Evolution of residual stresses}

Fig. 5 shows the evolution of longitudinal residual stresses $\sigma_{11}^{\exp }(\mathrm{hkil})\left(\varphi=0^{\circ}\right)$ calculated using equation (6) as a function of position $z$ and the total deformation for the different selected planes. The residual stresses on the undeformed sample are weak: $-22(17) \mathrm{MPa}$ for the $\{0004\}$ plane, 23 (6) $\mathrm{MPa}$ for the $\{20 \overline{2} 2\}$ plane and 73 (3) MPa for the $\{20 \overline{2} 5\}$ plane. These results show that the initial mechanical state is relatively homogenous within the sample and the thermal residual stresses are assumed to be negligible in the theoretical investigations. As can be seen in these figures, the tubeforming operation can be divided into three principal steps:

(a) A particular evolution takes places in the first $30 \mathrm{~mm}$ of the reduction zone. The tube is rolled, without being reduced, by a mandrel-tube-die contact ('tube sinking area'). The XRD data shed light on this phenomenon. The analysed planes are in traction, with a stress value that increases with the amount of prestrain. At $z=17 \mathrm{~mm}$, the value of $\sigma_{11}$ (hkil) reaches 377 (12) MPa for the $\{0004\}$ plane and 421 (12) $\mathrm{MPa}$ for the $\{20 \overline{2} 5\}$ plane.

(b) From $z \simeq 30 \mathrm{~mm}$ to $z \simeq 100 \mathrm{~mm}$, the residual stresses for all planes (except for the $\{10 \overline{1} 4\}$ plane, which decreases only slightly) decrease quickly, in relation to the total deformation, down to a compressive state. For example, the most striking evolution is that of the $\{0004\}$ stress, which develops rapidly up to a very large magnitude [-562 (28) MPa]. Differences can be observed for the other planes: -140 (13) MPa for $\{2025\}$ and 53 (9) MPa for $\{10 \overline{1} 4\}$. These X-ray measurements show the effective existence of plastic anisotropy. As can be seen from equation (4), the analysed stress depends on a function of the analysed family of planes. Strain incompatibilities are present at the mesoscopic level in the material, and consequently the stresses obtained by XRD depend on the plane. The diffracting crystals are not the same in each case and this allows us to deduce that different and important second-order stresses exist, linked to a strong plastic anisotropic deformation for these plane families. As shown by the diffraction, this area, where maximum stress occurs, is probably the zone that makes the principal contribution to the damage, owing to the presence of significant plastic strain (Montmitonnet et al., 2002). During the cold-pilgering process, $70 \%$ of the thickness reduction is made in this particular zone (Girard, 1993; Girard et al., 2001).

(c) Beyond $z \simeq 100 \mathrm{~mm}$, stress variations are less important (see Fig. 5). At $z=275 \mathrm{~mm}$, the rolling process is ended by a 'calibration' step which ensures a circular section to the final tube without plastic strain. For the finished tube, we can note the variation of the stress values in relation to the analysed plane: the $\{10 \overline{1} 4\}$ plane is in traction with a value of 189 (16) MPa, while the others present compressive stresses varying from -174 (19) to -409 (13) $\mathrm{MPa}$. Contrary to cold rolling of sheets (Gloaguen et al., 2007), cold pilgering generates significant second-order stresses of plastic origin associated with lower macroscopic stresses.

While the analysed stresses determined by XRD can be interpreted qualitatively by simple inspection, a more quantitative interpretation of the influence of the intergranular stresses on the development of the analysed stress requires the application of a polycrystalline model. Furthermore, these experimental results provide a comprehensive data set to test the scale transition model developed for this study.

\section{Analyses of XRD results using the scale transition model}

The EPSC model is used to simulate the residual intergranular strains (or stresses) and the crystallographic texture after

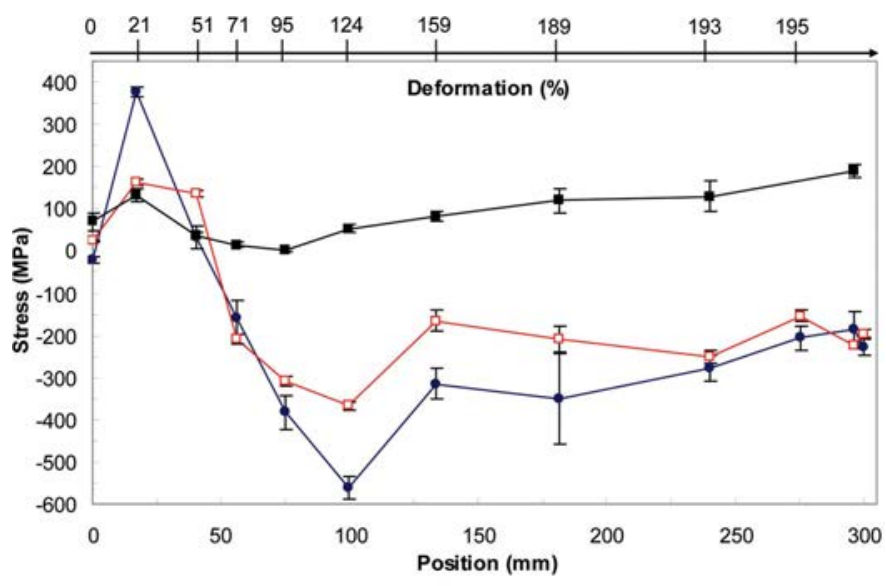

(a)

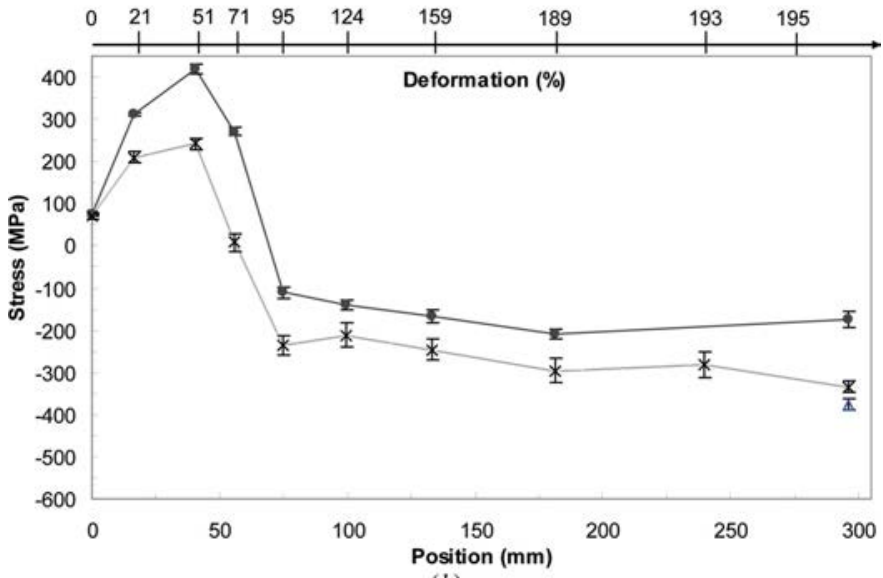

(b)

Figure 5

Longitudinal stress $\sigma_{11}^{\exp }(h k i l)$ calculated with the relation (4) $\left[\left\langle\varepsilon^{\text {IIplasticity }}(\varphi, \psi, h k i l)\right\rangle_{V_{\mathrm{d}}}=0\right]$ and obtained with different crystallographic planes. (a) Black squares denote data for $\{10 \overline{1} 4\}$, open squares for $\{20 \overline{2} 2\}$ and black circles for $\{0004\}$ (with Cr radiation). (b) Black circles denote data for $\{20 \overline{2} 5\}$, the open triangle for $\{30 \overline{3} 2\}$ and crosses for $\{21 \overline{3} 3\}$ (with $\mathrm{Cu}$ radiation). Error values were determined from the standard deviations of the peak positions as obtained from peak fitting. 
Table 1

Material parameters used in modelling.

Symbols are as defined in the text.

\begin{tabular}{llll}
\hline$\tau^{\mathrm{pr}\langle a\rangle}(\mathrm{MPa})$ & $\tau^{\mathrm{pyr}\langle a\rangle}(\mathrm{MPa})$ & $\tau^{\mathrm{pyr}\langle c+a\rangle}(\mathrm{MPa})$ & $\tau^{\mathrm{ttw}}(\mathrm{MPa})$ \\
\hline 95 & 175 & 310 & 180 \\
\hline$H^{\mathrm{pr}\langle a\rangle}(\mathrm{MPa})$ & $H^{\mathrm{pyr}\langle a\rangle}(\mathrm{MPa})$ & $H^{\mathrm{pyr}\langle c+a\rangle}(\mathrm{MPa})$ & $H^{\mathrm{ttw}}(\mathrm{MPa})$ \\
\hline 80 & 170 & 200 & 180 \\
\hline
\end{tabular}

\begin{tabular}{lll}
\hline$K_{0}$ & $K_{1}$ & $\beta\left(\mathrm{MPa}^{-1}\right)$ \\
\hline 25 & 1 & $1 / H^{\mathrm{rr}}$ \\
\hline
\end{tabular}

loading along the RD. The mesoscopic stresses are then averaged and projected on the $\varphi \psi$ directions to simulate the behaviour of the diffracting volume, and the simulated crystallographic reorientation is also obtained. For this study, a comparison between simulations and experiment has only been made for the finished tube. Although the scale transition models can reproduce the main features of this kind of process (Lebensohn et al., 1992; Girard et al., 2001), the details of the stress pattern can be determined only by the finite element method, owing to the geometric complexity.

Based on different experimental observations (Akhtar, 1973a,b, 1975; Tenckhoff, 1988; Pochettino et al., 1992), the deformation systems in the model are assumed to be the following: prismatic slip $\{10 \overline{1} 0\}\langle 11 \overline{2} 0\rangle \quad(\operatorname{pr}\langle a\rangle)$, first-order pyramidal slips $\{10 \overline{1} 1\}\langle 11 \overline{2} 3\rangle$ and $\{10 \overline{1} 1\}\langle 11 \overline{2} 0\rangle(\operatorname{pyr}\langle c+a\rangle$ and pyr $\langle a\rangle)$, basal slip $\{0001\}\langle 11 \overline{2} 0\rangle$ (b), and tensile twinning $\{10 \overline{1} 2\}\langle 10 \overline{1} 1\rangle$ (ttw). In the model, CRSS and the hardening law for slip and twinning are considered as controlling parameters. Their values depend on many factors (oxygen content, grain size, temperature etc.; Kaschner \& Gray, 2000) and are unknown. They were taken as adjustable fitting parameters. The initial CRSSs are taken to be identical for all systems of a system type.

To reduce the number of fitting parameters, we consider a linear hardening law (Gloaguen et al., 2007) where the coefficient $H^{t r}$ is equal to $H^{t t}$ for any deformation modes $r$ : $\dot{\boldsymbol{\tau}}^{t}=H^{t t} \sum_{r} \dot{\gamma}^{r}$, i.e. latent hardening is equal to self-hardening. As a result, the number of initial fitting parameters is reduced to 13. Comparisons between the model and the diffraction data have been made, considering six planes for the residual strains and three for the texture.

In the first step, the effect of deformation modes on the predicted strain (and stress) and texture was systematically analysed. Each deformation mode was applied independently within the EPSC model, in order to observe its effect on the strain accumulation and the texture. The effect of combining two or three systems was also examined. The values and signs of the predicted strains differed much more than those detected experimentally. The observed trends and magnitudes of the texture and strains were not reproduced correctly by the model in the case of one or two deformation modes. Finally, all five deformation systems were applied simultaneously with different sets of material parameters (CRSS, hardening matrix). Basal slip was not included in the set of deformation modes because some discrepancies appear with the X-ray results if this slip system is an active deformation mode. The best agreement was found with the values given in Table 1. The final choice of $\operatorname{pyr}\langle a\rangle$ as the active system is not selfevident: there is no conclusive evidence for the occurrence of pyramidal slip pyr $\langle a\rangle$ under any circumstances [see Xu et al. (2008) for more details]. Nevertheless, it is possible to explain and reproduce the texture without the $\operatorname{pyr}\langle a\rangle$ system. However, the model cannot correctly simulate the experimental residual strains. Only these four systems with $\operatorname{pr}\langle a\rangle$ as the main deformation mode can explain each experimental result.

The predicted PF for the final tube are given in Fig. 6. As shown in this figure, there is agreement between the experimental results and the EPSC model for the three selected FP. The main features of experimental texture are reproduced by the simulations. We clearly see predicted basal poles preferentially oriented at $38^{\circ}$ from RD in the RD-TD plane. With the CRSS values chosen above, deformation is accommodated through $\operatorname{pr}\langle a\rangle$ and $\operatorname{pyr}\langle c+a\rangle$. The relative contribution of ttw is small. At $195 \%$ deformation, the proportions of the activated systems are $57 \%$ for prismatic mode, $35.2 \%$ for pyramidal slips $\langle c+a\rangle, 7.8 \%$ for $\operatorname{pyr}\langle a\rangle$ and $0 \%$ for tensile twinning (it reaches a maximum value of $1.5 \%$ at $12 \%$ total deformation). Finally, we obtain a reasonable distribution in agreement with the experimental observations: prismatic glide is the main active mechanism, pyramidal slip is a secondary slip mode and twinning contributes very little to the plastic deformation. The weak activation of the tensile twinning is normal because it preferentially reorientates those grains with $c$ axes close to the RD.

The theoretical values of the $\left\langle\varepsilon^{\mathrm{IIth}}(\varphi, \psi, h k i l)\right\rangle_{V_{\mathrm{d}}}$ strain were also predicted by the EPSC model for $195 \%$ strain. Applying equation (4) and fitting the results obtained from the model to the experimental data obtained from the six crystallographic planes, the first-order stress tensor element $\sigma_{11}^{\mathrm{I}}$ and the $q$ factor were determined using the least-squares method. The same set of material parameters was used for this confrontation. The predicted results agree with the fact that, in the $\langle\varepsilon(\varphi, \psi, h k i l)\rangle_{V_{\mathrm{d}}}$ versus $\sin ^{2} \psi$ representation of the deformed samples, the best fit for the six studied planes is obtained with prismatic slip as the main deformation mode. The results of the analyses on the final tube are given in Table 2. With the help of the ODF, it is possible to find the set of principal orientations that contribute to the diffracting volume for the
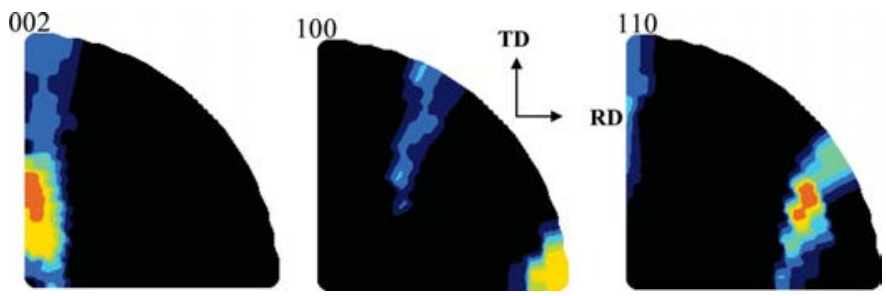

Figure 6

Predicted PF $\{0002\},\{10 \overline{1} 0\}$ and $\{11 \overline{2} 0\}$ after the forming process. 
different $\psi$ values (between -48 and $45^{\circ}$ ) and for each plane after deformation. In Fig. 7, the $c$ axis of these orientations for the planes $\{10 \overline{1} 4\},\{30 \overline{3} 2\}$ and $\{21 \overline{3} 3\}$ is plotted in a $\{0002\}$ pole figure. For example, because of the final texture, most of the $\{10 \overline{1} 4\}$ planes have the $c$ axis making an angle of between 0 and $25^{\circ}$ with the sample surface, whereas this axis is preferentially located between 50 and $70^{\circ}$ in relation to the surface for the $\{30 \overline{3} 2\}$ planes. Because of the second-order stresses, the obtained residual stress values are not directly related to those obtained by macroscopic methods (Girard, 1993) and depend strongly on the analysed plane.

In order to show the influence of second-order strain of plastic origin, Table 2 gives the first-order stress calculated with and without the $\left\langle\varepsilon^{\text {IIplasticity }}(\varphi, \psi, h k i l)\right\rangle_{V_{\mathrm{d}}}$ term. $\sigma_{11}^{\mathrm{I}}$ is 60 (30) $\mathrm{MPa}$. If the second-order 'plastic' stresses are presumed to be null $\left[\langle\varepsilon(\varphi, \psi, h k i l)\rangle_{V_{\mathrm{d}}}=F_{i j}(\varphi, \psi, h k i l) \sigma_{i j}^{\exp }(h k i l)\right]$, the 'experimental' stresses are then $\sigma_{11}^{\exp }(10 \overline{1} 4)=189$ (16) MPa and $\sigma_{11}^{\exp }(21 \overline{3} 3)=-334$ (6) MPa along the RD. This example allows us to conclude clearly that the $\left\langle\varepsilon^{\text {IIplasticity }}(\varphi, \psi, h k i l)\right\rangle_{V_{\mathrm{d}}}$ term plays a key role in the correct interpretation of the X-ray diffraction results. As can be seen in Fig. 7, the sets of grains that contribute to the diffracting volume for each plane are not the same. Consequently, the plastic activity and the active deformation systems differ between these two sets of diffracting grains, this difference being linked to the orientation dependence of the plastic deformation. At $195 \%$ total strain, the calculated relative activities of the deformation modes for the $\{10 \overline{1} 4\}$ and $\{30 \overline{3} 2\}$ grains, respectively, are 64.6 and $47.5 \%$ for prismatic mode, 35.4 and $35.0 \%$ for pyramidal slips $\langle c+a\rangle, \sim 0$ and $17.5 \%$ for $\operatorname{pyr}\langle a\rangle$, and 0 and $0 \%$ for tensile twinning. $\operatorname{Pyr}\langle a\rangle$ is more active for the $\{30 \overline{3} 2\}$ grains, whereas

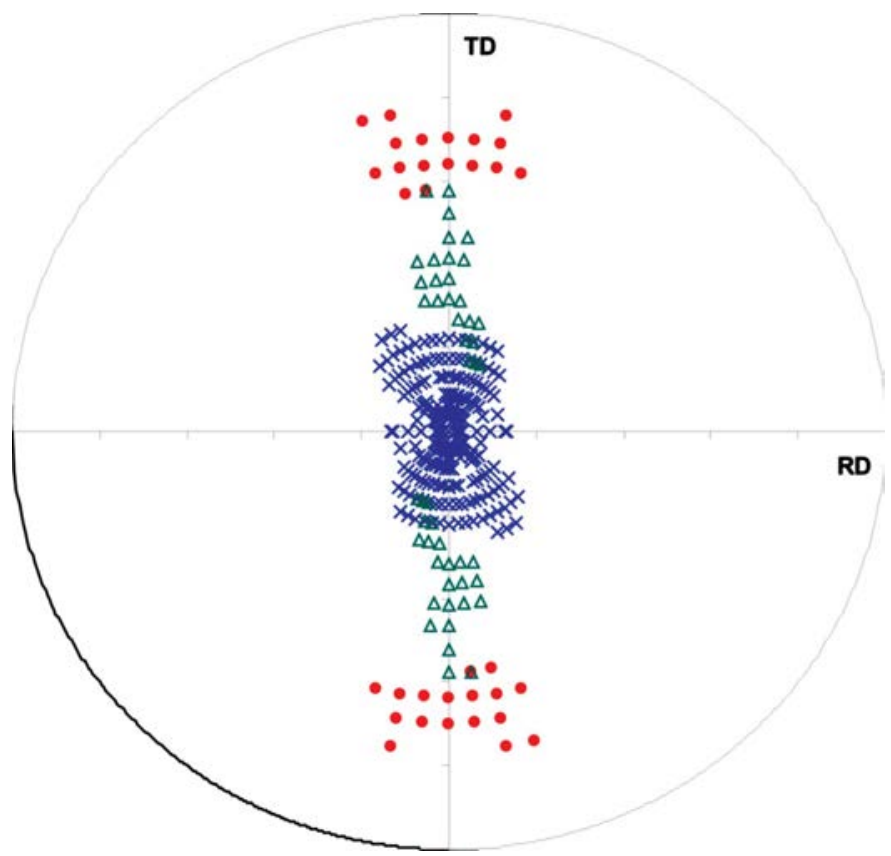

Figure 7

The set of principal orientations that contribute to the diffracting volume for the different $\psi$ values (between -48 and $45^{\circ}$ ) and for the planes $\{10 \overline{1} 4\}$ (crosses), $\{30 \overline{3} 2\}$ (solid circles) and $\{21 \overline{3} 3\}$ (open triangles) plotted in a $\{0002\}$ pole figure after deformation.
Table 2

Comparison of the stress values determined with two assumptions, i.e. $\left\langle\varepsilon^{\text {Iplasticity }}(\varphi, \psi, h k i l)\right\rangle_{V_{\mathrm{d}}} \neq 0$ and $\left\langle\varepsilon^{\text {Iplasticity }}(\varphi, \psi, h k i l)\right\rangle_{V_{\mathrm{d}}}=0$.

\begin{tabular}{llll}
\hline & $q$ & $\sigma_{11}^{\text {I }}(\mathrm{MPa})$ & \\
\hline$\left\langle\varepsilon^{\text {IIplasticity }}(\varphi, \psi, h k i l)\right\rangle_{V_{\mathrm{d}}} \neq 0$ & $0.202(81)$ & $60(30)$ & \\
$\left\langle\varepsilon^{\text {IIplasticity }}(\varphi, \psi, h k i l)\right\rangle_{V_{\mathrm{d}}}=0$ & - & $\{10 \overline{1} 4\}$ plane & $\{30 \overline{3} 2\}$ plane \\
& & $189(16)$ & $-376(14)$ \\
\hline
\end{tabular}

its mean contribution to the deformation is much smaller for all the grains and almost negligible for the $\{10 \overline{1} 4\}$ grains. Furthermore, the global stress and strain tensors for each set are different. For example, the $\{10 \overline{1} 4\}$ grains have a higher mean strain (with a lower stress) than the $\{30 \overline{3} 2\}$ grains. A ratio of nearly 1.5 is calculated at $195 \%$ deformation on comparing the strain components along the rolling direction in these two sets of grains. These differences are related to the plastic anisotropy, which depends heavily on the crystallographic orientation.

To finish, we note that the reliability of the $q$ values seems to be correct compared with previous work on cubic materials (Baczmanski et al., 1994, 2008). This can be explained as the overprediction of residual stresses by the self-consistent model. It is a well known feature of this model (Krier, 1993; Masson et al., 2000; Zaoui \& Masson, 2000), which is too 'stiff' because it does not take into account the fluctuations of moduli in the matrix or inclusions due to the nonlinearity of the mechanical behaviour. Elastoplastic properties are considered to be uniform inside the crystallites. The formation of a particular microstructure (dislocation band, wall etc.) inside a crystal is neglected. Discontinuities (grain boundaries, dislocation walls etc.) are assumed to have a weak influence on the average behaviour of the aggregate, and dislocation annihilations are not taken into account (Mabelly et al., 1996; Gloaguen \& François, 2006; Fajoui et al., 2009). Nevertheless, the model allows us to describe the evolution of residual stresses whatever the plane and enables us to determine the macroscopic stress. This behaviour can only be explained with prismatic slip as the main deformation mode and for the same set of material parameters. For h.c.p. alloys, the value of the second-order stress is important after a large plastic deformation.

\section{Conclusions}

Analysis of texture and residual stress using X-ray diffraction has been carried out on $\mathrm{Zr}$ alloy cladding tubes after an industrial mechanical process, cold pilgering. The last rolling pass has been completely characterized by X-ray diffraction in the transition area between the raw tube and the finished one. A quantitative interpretation of the influence of intergranular stresses on the development of analysed stress has been performed using a modified elastoplastic self-consistent polycrystalline model. The experimental results provided a comprehensive data set to test the scale transition model developed for this study. The present study highlights the usefulness of a polycrystal model for exploring the active 
deformation modes in h.c.p. alloys. Reasonable agreement between model predictions and experimental measurements was observed for both residual stresses and evolved deformation textures. The stresses measured by X-ray diffraction differ significantly from one lattice plane family to another. These results can be explained by the presence of first- and second-order stresses of plastic origin. The choice of prismatic slip as the main deformation mode can explain the experimental texture and stress values for the six studied planes. The influence of second-order strain linked to the strong plastic anisotropy of this type of alloy must be taken into account in order to obtain a correct interpretation of the X-ray diffraction results.

\section{References}

Akhtar, A. (1973a). Acta Metall. 21, 1-11.

Akhtar, A. (1973b). J. Nucl. Mater. 47, 79-86.

Akhtar, A. (1975). Metall. Trans. A, 6, 1105-1113.

Baczmanski, A., Hfaiedh, N., François, M. \& Wierzbanowski, K. (2009). Mater. Sci. Eng. A, 501, 153-165.

Baczmanski, A., Lipinski, P., Tidu, A., Wierzbanowski, K. \& Pathiraj, B. (2008). J. Appl. Cryst. 41, 854-867.

Baczmanski, A., Wierzbanowski, K., Lipinski, P., Helmholdt, R. B., Ekambaranathan, G. \& Pathiraj, B. (1994). Philos. Mag. A, 69, 437449.

Castelnau, O., Francillette, H., Bacroix, B. \& Lebensohn, R. A. (2001). J. Nucl. Mater. 297, 14-26.

Clausen, B., Lorentzen, T. \& Leffers, T. (1998). Acta Mater. 46, 30873098.

Dionnet, B., François, M., Sprauel, J. M. \& Nardou, F. (1999). J. Appl. Cryst. 32, 883-891.

Dölle, H. (1979). J. Appl. Cryst. 12, 489-501.

Fajoui, J., Gloaguen, D., Courant, B. \& Guillén, R. (2009). Comput. Mech. 44, 285-296.

Franz, G., Abed-Meraim, F., Ben Zineb, T., Lemoine, X. \& Berveiller, M. (2009). Comput. Mater. Sci. 45, 768-773.

Girard, E. (1993). PhD thesis, University of Nantes, France.

Girard, E., Guillén, R., Weisbecker, P. \& François, M. (2001). J. Nucl. Mater. 294, 330-338.

Gloaguen, D., Berchi, T., Girard, E. \& Guillén, R. (2006). Phys. Status Solidi A, 203, 12-14.

Gloaguen, D., Berchi, T., Girard, E. \& Guillén, R. (2007). Acta Mater. 55, 4369-4379.

Gloaguen, D. \& François, M. (2006). Phys. Status Solidi A, 203, 19401953.

Gloaguen, D., François, M. \& Guillen, R. (2004). J. Appl. Cryst. 37, 934-940.

Guillén, R., Cossu, C., François, M. \& Girard, E. (1998). J. Nucl. Mater. 255, 174-179.
Guillén, R., Cossu, C., Jacquot, T., François, M. \& Bourniquel, B. (1999). J. Appl. Cryst. 32, 387-392.

Guinebretière, R. (2007). X-ray Diffraction by Polycrystalline Materials. Washington, DC: ISTE.

Hauk, V. (1997). Structural and Residual Stress Analysis by Nondestructive Methods. Amsterdam: Elsevier.

Hill, R. (1965). J. Mech. Phys. Solids, 13, 89-105.

Hutchinson, W. (1970). Proc. R. Soc. London Ser. A, 319, 247-272.

Kapoor, K., Lahir, D., Padmaprabu, C. \& Sanyal, T. (2002). J. Nucl. Mater. 303, 147-155.

Kaschner, G. C. \& Gray, G. T. (2000). Metal. Mater. Trans. A, 31, 1997-2033.

Kaschner, G. C., Tomé, C. N., Beyerlein, I. J., Vogel, S. C., Brown, D. W. \& McCabe, R. J. (2006). Acta Mater. 54, 2887-2896.

Kiran Kumar, M., Vanitha, C., Samajadr, I., Dey, G. K., Tewari, R., Srivastava, D. \& Banerjee, S. (2004). J. Nucl. Mater. 335, 48-58.

Krier, J. (1993). PhD thesis, University of Metz, France.

Kröner, E. (1961). Acta Metall. 9, 155-165.

Lebensohn, R. A., Gonzalez, M. I., Tomé, C. N. \& Pochettino, C. A. (1992). J. Nucl. Mater. 229, 57-64.

Linga Murty, K. \& Charit, I. (2006). Prog. Nucl. Energy, 48, 325-359.

Lipinski, P. \& Berveiller, M. (1989). Int. J. Plast. 5, 149-172.

Lorrain, J. P., Ben Zineb, T., Abed-Meraim, F. \& Berveiller, M. (2005). Int. J. Forming Process. 8, 135-158.

Mabelly, P., Hadmar, P., Desvignes, M. \& Sprauel, J. M. (1996). Proc. Fourth Eur. Conf. Residual Stresses, 2, 941-949.

Macherauch, E., Wohlfarhrt, H. \& Wolfstieg, U. (1973). Härterei Tech. Mitt. 28, 201-211.

Masson, R., Bornert, M., Suquet, F. \& Zaoui, A. (2000). J. Mech. Phys. Solids, 48, 1203-1277.

Matthies, S., Priesmeyer, H. G. \& Daymond, M. R. (2001). J. Appl. Cryst. 34, 585-601.

Montmitonnet, P., Logé, R., Hamery, M., Chastel, Y., Doudoux, J. L. \& Aubin, J. L. (2002). J. Mater. Process. Technol. 125-126, 814-820.

Noyan, I. C. \& Cohen, J. B. (1987). Residual Stress - Measurements by Diffraction and Interpretation. Heidelberg: Springer-Verlag.

Ortiz, M. \& Pochettino, A. A. (1996a). J. Nucl. Mater. 229, 65-72.

Ortiz, M. \& Pochettino, A. A. (1996b). Proc. Fourth Eur. Conf. Residual Stresses, 2, 971-976.

Pochettino, A. A., Gannio, N., Vial Edwards, C. \& Penelle, R. (1992). Scr. Metall. 27, 1859-1863.

Proust, G., Tomé, C. N. \& Kaschner, G. C. (2007). Acta Mater. 55, 2137-2148.

Tenckhoff, E. (1988). Editor. Deformation Mechanisms, Texture and Anisotropy in Zirconium and Zircaloy. Philadelphia: ASTM International.

Tomé, C. N., Lebensohn, R. A. \& Kocks, U. F. (1991). Acta Metall. Mater. 11, 2667-2680.

Wenk, H.-R., Matthies, S., Donovan, J. \& Chateigner, D. (1998). J. Appl. Cryst. 31, 262-269.

Xu, F., Holt, R. A. \& Daymond, M. R. (2008). Acta Mater. 56, 3672 3687.

Zaoui, A. \& Masson, R. (2000). Mater. Sci. Eng. A, 285, 418-424. 